\title{
Erratum to: Application of Next-Generation Sequencing to Hearing Loss
}

\author{
Lisa Dyer, Xinjian Wang, Kejian Zhang, John Greinwald Jr.,
} and C. Alexander Valencia

\author{
Erratum to: \\ Chapter 5 in: L.-J.C. Wong (ed.), Next Generation Sequencing \\ Based Clinical Molecular Diagnosis of Human Genetic Disorders, \\ DOI 10.1007/978-3-319-56418-0_5
}

In chapter titled "Application of Next-Generation Sequencing to Hearing Loss", the authors realized that the first author of the chapter should be Lisa Dyer not Xinjian Wang. Xinjian Wang should be second author. The correction has been updated in the chapter now. 